\title{
Cultivares e portaenxertos sobre o vigor de plantas de pereira europeias
}

\author{
Cultivars and rootstocks on plants vigor of European pear
}

\author{
Bruno Dalazen Machado ${ }^{\mathrm{I}}$ Leo Rufato ${ }^{\mathrm{I}}$ Amauri Bogi ${ }^{*}{ }^{*}$ Aike Anneliese Kretzschmar $^{\mathrm{I}}$ \\ André Emmel Mario ${ }^{I}$
}

\section{RESUMO}

A cultura da pereira (Pyrus communis) possui grande potencial de expansão no sul do Brasil devido às condições climáticas e solo. A carência de informações a respeito da(s) melhor(es) combinação(ões) de cultivares copa de pereira europeia e portaenxertos, quanto ao aspecto vegetativo e potencial produtivo, tem limitado o cultivo. O objetivo do trabalho foi avaliar a influência de cultivares copa de pereira europeia e portaenxertos de marmeleiro (Cydonia oblonga) sobre o vigor e variáveis de plantas. Os experimentos foram conduzidos em pomares comerciais da cidade de Fraiburgo, Estado de Santa Catarina, durante os ciclos de cultivo 2008/09, 09/10 e 10/11. As cultivares avaliadas foram 'Packham's Triumph', 'Santa Maria', 'Rocha'e 'Abbè Fetel' e os portaenxertos de marmeleiro EMC e Adams. O espaçamento de plantio foi de 0,3m entre plantas e $4 m$ entre linhas. As variáveis analisadas foram: a. incremento de altura de plantas $(\mathrm{m})$; $b$. incremento de volume de copa $\left(\mathrm{m}^{3}\right)$; c. incremento de diâmetro do tronco da cv. 'copa' $(\mathrm{mm}) ; \mathrm{d}$. incremento de diâmetro do tronco do portaenxerto ( $\mathrm{mm}$ ); e. diferença de diâmetro do tronco entre a cultivar copa e o portaenxerto ( $\mathrm{mm}) ; \mathrm{f}$. índice de fertilidade ( $\mathrm{n}^{\circ}$ de gemas $\mathrm{cm}^{-1}$ ); g. massa fresca média total acumulada do material vegetativo das três podas de inverno $(\mathrm{kg}) ; \mathrm{h}$. diferença cumulativa entre a massa fresca e a massa seca dos ramos podados $(\mathrm{kg})$. As diferentes combinações de cultivares e portaenxertos interferiram nas diferentes variáveis analisadas. As combinações Abbè Fetel e Rocha sobre marmelo Adams, foram significativamente mais vigorosas em termos de incremento de diâmetro do tronco da cultivar copa, incremento de altura de plantas, incremento de volume de copa e massa fresca média total acumulada do material vegetativo das podas de inverno dos três anos. A combinação Santa Maria sobre marmelo Adams apresentou vigor intermediário significativo de plantas de acordo com o incremento em volume de copa, diferença de diâmetro do tronco entre a cultivar copa e do portaenxerto, sendo mais indicada para uso comercial, devido ao maior equilíbrio proporcionado entre parte vegetativa e produtiva da planta, nas condições edafoclimáticas experimentais do Sul do Brasil.

Palavras-chave: Pyrus communis L., Cydonia oblonga L., variáveis.

\section{ABSTRACT}

The pear (Pyrus communis) culture offers a great expansive market opportunity in southern Brazil, according to climatic conditions and soil. However, there are still some barriers that prevent satisfactory economic production, such as lack of knowledge about the best combinations of cultivars and rootstocks. The objective of the study was to evaluate the influence of European pear cultivars and quince (Cydonia oblonga) rootstocks on the plants vigor variables. The experiments were carried out in commercials orchards in Fraiburgo Municipality, Santa Catarina state, during the crop season of 2008/09, 09/10 and 10/11. The cultivars evaluated were 'Packham's Triumph', 'Santa Maria', 'Rocha' and 'Abbè Fetel' and the quince rootstocks EMC and Adams. The cultivars and rootstocks combinations were conducted in a spacing of $0.3 \mathrm{mx} 4 \mathrm{~m}$ between plants and rows, respectively. The vigor variables evaluated were, $a$. plants height increment $(m)$; b. canopy volume increment $\left(\mathrm{m}^{3}\right)$; c. truck diameter increment of the cultivars canopy $(\mathrm{mm}) ; d$. truck diameter increment of rootstocks ( $\mathrm{mm}) ;$ e. difference between the trunk diameter of cultivars canopy and rootstock $(\mathrm{mm})$; $f$. fertility index (buds $\mathrm{cm}^{-1}$ ); $\mathrm{g}$. total cumulative average of fresh weight of pruned branches ( $\mathrm{kg})$; and $\mathrm{h}$. cumulative difference between the fresh and dry weight of pruned branches $(\mathrm{kg})$. All combinations evaluated affected the vegetative parameters of the European pear cultivars. The combinations Abbè Fetel and Rocha grafted on Adams quince rootstocks are significant more vigorous according to increment of cultivars canopy truck diameter, plant height increment, cultivars canopy volume increment and cumulative average weight of pruned branches, when compared with others combinations. The combination Santa Maria grafted on Adams quince rootstock showed significant intermediate vigor according to cultivar canopy volume increment, truck diameter of cultivar canopy and, truck diameter of rootstock. This combination presented a better proportion amongst the vigor variables and can be indicated for commercial production according to the experimental edafoclimatic conditions of southern Brazil.

Key words: Pyrus communis L., Cydonia oblonga L., variables.

'Programa de Pós-graduação em Produção Vegetal, Centro de Ciências Agroveterinárias (CAV), Universidade do Estado de Santa Catarina (UDESC), Av. Luis de Camões, 2009, 88520-000, Lages, SC, Brasil. E-mail: amauribogo@udesc.br. *Autor para correspondência. Recebido 10.06.12 Aprovado 09.04.13 Devolvido pelo autor 25.06.13 


\section{INTRODUÇÃO}

Relacionando a produção brasileira de pera (Pyrus communis L.) com seu consumo, observa-se que a cultura apresenta grande potencial de expansão, principalmente no sul do país, onde existem condições climáticas e de solo favoráveis, tornandose uma alternativa consistente para a diversificação da fruticultura de clima temperado nessa região. Um dos fatores limitantes de produções economicamente satisfatórias está na limitação de cultivares e portaenxertos adaptados às diferentes regiões potencialmente produtoras. Grande parte das áreas implantadas de pereira no Brasil estão enxertadas sobre o portaenxerto P.calleryana (Decne.), o qual proporciona às plantas alto vigor e lento período de entrada em produção (GIACOBBO et. al., 2007).

$\mathrm{O}$ vigor é uma boa medida do desempenho de uma planta e existem várias variáveis que podem estimar o vigor (HARTMANN et al., 2002; SHAFFER et al., 2004; TOMAZ et al., 2009). No atual sistema de produção, com a utilização de portaenxertos vigorosos, uma cultivar de pereira europeia leva em média de seis a sete anos para entrar em produção, devido ao alto vigor conferido às plantas pelo portaenxerto, o que retarda a entrada em frutificação e dificulta as práticas de manejo, inviabilizando, dessa forma, qualquer agronegócio (PIO et al., 2008). Com o surgimento de diferentes clones de marmeleiro (Cydonia oblonga L.), iniciouse uma nova fase de produção de pera, caracterizada por menor vigor e uniformidade de produção (QUEZADA et al., 2003). Os marmeleiros vêm sendo utilizados como portaenxertos preferenciais para a pereira, com o intuito de proporcionar plantas de pequeno porte e rápida frutificação, além de conferir uniformidade aos pomares (PIO et al., 2008; STERN \& DARON, 2009; MILOSEVIC \& MILOSEVIC, 2011). No entanto, existem poucas informações sobre quais tipos de marmeleiros que podem ser usados como portaenxertos para as cultivares europeias de pereira, assim como a sua relação no controle do vigor das plantas e a respectiva melhoria na eficiência produtiva da pereira. Dentre os fatores para que a cultura da pereira tenha maior expressão, está à necessidade de definir as melhores combinações de cultivares copa e portaenxertos mais adaptados às regiões potencialmente produtoras. $\mathrm{O}$ objetivo deste trabalho foi avaliar a influência de cultivares copa de pereira europeia e portaenxertos de marmeleiro sobre o vigor de plantas nas condições edafoclimáticas de Fraiburgo, estado de Santa Catarina, durante os ciclos de cultivo 2008/09, 09/10 e 10/2011.

\section{MATERIAL E MÉTODOS}

Os experimentos foram conduzidos em pomares comerciais de pereira, localizados em Fraiburgo no estado de Santa Catarina $\left(27^{\circ} 01\right.$ 's e $\left.50^{\circ} 55^{\prime} \mathrm{W}\right)$. A região está localizada a aproximadamente $1048 \mathrm{~m}$ de altitude acima do nível do mar, com o clima tipo mesotérmico úmido (Cfb), de acordo com a classificação de Köppen. Alta precipitação ocorre de outubro a março e, durante esse período, as médias mensais de chuva são de aproximadamente $224,1 \mathrm{~mm}$. O solo é classificado como um Nitossolo com textura franco argilosa, com teores altos de matéria orgânica, fósforo e potássio.

Durante os ciclos de cultivo 2008/09, 09/10 e 10/11, a precipitação anual e umidade relativa média do ar foram de 1910,1, 1887,2 e 1283,8mm e, $74,8 \%, 75,5 \%$ e $88,6 \%$, respectivamente (EPAGRI, 2011). O número de unidades de frio, segundo o Método Carolina do Norte Modificado (EBERT et al., 1986), foi em média de 450 para os ciclos de cultivo entre os meses de maio a setembro.

A área experimental foi implantada em agosto de 2007, com espaçamento de 0,3m entre plantas e 4,0m entre linhas, correspondendo a uma densidade de plantio de 7500 plantas $\mathrm{ha}^{-1}$. O plantio foi realizado com mudas pré-formadas e o sistema de condução adotado foi o Líder Central. Os tratos culturais foram semelhantes para todos os experimentos: adubações com base na análise de solo; arqueamento de ramos; tratamentos fitossanitários e controle de plantas daninhas quando necessários; e irrigação por gotejamento.

As combinações utilizadas de cultivares copa enxertadas sobre portaenxertos (cultivar/ portaenxerto) foram: 'Rocha'/Adams, 'Rocha'/EMC, 'Santa Maria'/Adams, 'Packham's Triumph'/EMC, 'Abbè Fetel'/EMC e 'Abbè Fetel'/Adams.

As variáveis analisadas foram: a. incremento de altura de plantas (m), com o auxílio de uma fita métrica a partir do solo até o ápice da planta. A primeira, segunda e terceira medições foram realizadas com as plantas em repouso vegetativo no inverno de 2009, 2010 e 2011; b. Incremento de volume de copa $\left(\mathrm{m}^{3}\right)$, medindo a altura $(\mathrm{m})$ da copa (H) a partir do ponto de inserção do primeiro ramo no tronco, a largura (L) (m) e a espessura (E) da copa (m) com o auxílio de uma régua de madeira com $2 \mathrm{~m}$ de comprimento, determinando, assim, o volume de copa através da fórmula $(\mathrm{LxExH})$; c. incremento de diâmetro do tronco da cultivar-copa (C) e do portaenxerto (PE) $(\mathrm{mm})$, medindo as plantas $5 \mathrm{~cm}$ abaixo e acima do ponto de enxertia com paquímetro digital. Calculou- 
se a diferença de diâmetro entre o portaenxerto e a cultivar-copa $(\mathrm{mm})$; d. Índice de fertilidade $\left(\mathrm{n}^{\circ}\right.$ de gemas $\mathrm{cm}^{-1}$ ), pela medição de três ramos de ano por planta com auxílio de fita métrica e contando o número de gemas (vegetativas e floríferas) por ramos. Pela relação número de gemas pelo comprimento do ramo, estimou-se o índice de fertilidade; e. Massa fresca média total acumulada $(\mathrm{Kg})$ do material vegetativo das três podas de inverno realizadas e pesadas através de balança digital; f. diferença cumulativa entre a massa fresca e a massa seca dos ramos podados através da retirada de uma amostra de ramos de cada poda. As amostras foram secas em estufa a $60^{\circ} \mathrm{C}$ por 4 dias para obter a diferença entre a massa seca e a massa fresca. Os dados das variáveis analisadas durante os três ciclos vegetativos foram apresentados de forma cumulativa e o delineamento experimental utilizado foi o de blocos ao acaso, com três repetições e dez plantas por parcela.

\section{RESULTADOS E DISCUSSÃO}

As médias acumuladas das variáveis analisadas de incremento de altura de plantas (IAP), incremento de volume de copa (IVC), índice de fertilidade de gemas (IFG), incremento no diâmetro do tronco da cv. 'copa' (IDC), incremento no diâmetro do tronco do portaenxerto (IDPE), diferença de diâmetro entre o portaenxerto e a cV. 'copa' (PE-C), massa fresca média total acumulada do material vegetativo das podas de inverno dos três anos (MFCP) e diferença entre a massa fresca e seca acumulada dos ramos podados dos três anos (DMFS), nos três ciclos de cultivo avaliados são apresentadas na tabela 1 .
Quando as variáveis IAP, IVC e IFG foram utilizadas para caracterizar o vigor das plantas, as combinações Abbè Fetel e Rocha/ Adams mostraram diferença significativa das demais combinações analisadas (Tabela 1). Essas combinações apresentaram maior desenvolvimento vegetativo e, consequente maior vigor, corroborando os resultados encontrados por TOMAZ et al. (2009), que utilizaram o IAP, IVC como variáveis de vigor de plantas. Segundo HARTMANN et al. (2002), o menor IFG deve-se ao maior crescimento vegetativo de planta, afetando o balanço entre a parte vegetativa e reprodutiva como resposta à maior translocação ascendente e descendente da seiva.

Durante os três ciclos de cultivo avaliados, foi observado que a combinação Abbè Fetel/Adams apresentou maior vigor de plantas que a combinação Rocha/Adams, com diferenças significativas no IDC, MFCP e DMFS, quando consideradas essas variáveis como padrões de vigor de plantas (Tabela 1). SHAFFER et al. (2004) observaram o mesmo comportamento quando relataram as diferenças dos portaenxertos em relação ao vigor conferido às plantas, necessitando de uma maior intervenção durante a poda de inverno, principalmente quando as plantas não foram submetidas à poda verde anteriormente.

Os maiores incrementos significativos de IDC e IDPE foram observados na combinação Abbè Fetel/Adams do que na combinação Abbè Fetel/EMC (Tabela 1). Esse maior vigor também foi atribuído pelo menor problema de incompatibilidade com o portaenxerto Adams, quando comparado com o portaenxerto EMC. Essa baixa incompatibilidade

Tabela 1 - Dados acumulados de variáveis de plantas para as diferentes combinações de cutivares copa de pereira europeia e portaenxertos de marmeleiro em Fraiburgo-SC, Brasil, durante as safras agrícolas de 2008/09, 09/10 e 10/11.

\begin{tabular}{|c|c|c|c|c|c|c|c|c|c|}
\hline Combinação & & $\begin{array}{l}\text { IAP } \\
(\mathrm{m})\end{array}$ & $\begin{array}{l}\text { IVC } \\
\left(\mathrm{m}^{3}\right)\end{array}$ & $\begin{array}{l}\text { IFG } \\
\left(\text { gemas cm }^{-1}\right)\end{array}$ & $\begin{array}{l}\text { IDC } \\
(\mathrm{mm})\end{array}$ & $\begin{array}{l}\text { IDPE } \\
(\mathrm{mm})\end{array}$ & $\begin{array}{l}\mathrm{PE}-\mathrm{C} \\
(\mathrm{mm})\end{array}$ & $\begin{array}{l}\text { MFCP } \\
(\mathrm{Kg})\end{array}$ & $\begin{array}{l}\text { DMFS } \\
(\mathrm{kg})\end{array}$ \\
\hline cultivar & portaenxerto & $2009 / 11$ & $2009 / 11$ & $2009 / 11$ & $2009 / 11$ & $2009 / 11$ & $2009 / 11$ & $2009 / 11$ & $2009 / 11$ \\
\hline 'Abbè Fetel' & EMC & $1,24 \mathrm{~b}$ & $0,82 \mathrm{~b}$ & $1,50 \mathrm{ab}$ & $15,1 \mathrm{~b}$ & $18,7 \mathrm{~b}$ & $12,4 \mathrm{a}$ & $1,35 \mathrm{c}$ & $0,137 \mathrm{a}$ \\
\hline 'Abbè Fetel' & Adams & $1,67 \mathrm{a}$ & $1,10 \mathrm{a}$ & $1,38 \mathrm{c}$ & $19,5 \mathrm{a}$ & $27,6 \mathrm{a}$ & $3,5 \mathrm{c}$ & $5,91 \mathrm{a}$ & $0,167 \mathrm{a}$ \\
\hline 'Rocha' & Adams & $1,64 \mathrm{a}$ & $1,08 \mathrm{a}$ & $1,41 \mathrm{c}$ & $15,2 \mathrm{~b}$ & $27,1 \mathrm{a}$ & $2,2 \mathrm{c}$ & $1,71 \mathrm{~b}$ & $0,082 \mathrm{~b}$ \\
\hline 'Rocha' & EMC & $1,36 \mathrm{~b}$ & $0,64 \mathrm{c}$ & $1,53 \mathrm{a}$ & $14,5 \mathrm{bc}$ & $16,7 \mathrm{c}$ & $7,7 \mathrm{~b}$ & $1,23 \mathrm{c}$ & $0,080 \mathrm{~b}$ \\
\hline 'Packham's Triumph' & EMC & $1,24 \mathrm{~b}$ & $0,24 \mathrm{~d}$ & $1,54 \mathrm{a}$ & $7,6 \mathrm{~d}$ & $9,0 \mathrm{e}$ & $11,2 \mathrm{a}$ & $0,49 \mathrm{c}$ & $0,052 \mathrm{~b}$ \\
\hline 'Santa Maria' & Adams & $1,26 \mathrm{~b}$ & $0,62 \mathrm{c}$ & $1,50 \mathrm{ab}$ & $12,6 \mathrm{c}$ & $15,1 \mathrm{~d}$ & $2,5 \mathrm{c}$ & $1,64 \mathrm{~b}$ & $0,157 \mathrm{a}$ \\
\hline CV $(\%)$ & & 9,8 & 13,4 & 4,2 & 9,1 & 4,8 & 24,9 & 25,9 & 7,9 \\
\hline
\end{tabular}

* Médias seguidas pela mesma letra na coluna não diferem estatisticamente entre si pelo teste de Duncan a 5\% de probabilidade de erro. IAP: incremento de altura de plantas; IVC: incremento de volume de copa; IFG: índice de fertilidade de gemas; IDC: incremento no diâmetro do tronco da cv. 'copa'; IDPE: incremento no diâmetro do tronco do portaenxerto; PE-C: diferença de diâmetro entre o portaenxerto e a cv. 'copa'; MFCP: massa fresca média total acumulada do material vegetativo das podas de inverno dos três anos; DMFS: diferença entre a massa fresca e seca cumulativa dos ramos podados dos três anos. 
também foi observada por SANSAVANI et al. (1997), em estudo de portaenxertos para a cv. 'Abbè Fetel'.

A combinação Packham’s Triumph/EMC, além de apresentar significativa redução do vigor em termos de IAP, IVC, IDPE e PE-C, apresentou maior IFG, quando comparado com a combinação Abbè Fetel/Adams (Tabela 1). Esses dados corroboram RUFATO et al. (2012), que relataram, em estudos de poda verde com a cultivar 'Abbè Fetel' sobre diferentes portaenxertos, que o portaenxerto EMC pode induzir menor vigor às plantas, devido, entre outros fatores, ao maior índice de fertilidade.

As combinações Abbè Fetel/EMC e Packham's Triumph/EMC apresentaram as maiores diferenças de PE-C, quando comparadas às demais combinações (Tabela 1). Plantas com sintomas de incompatibilidade apresentam as maiores diferenças de diâmetro da copa em relação ao portaenxerto, confirmando a afirmação de VALLI (2002), a qual definiu, como sintoma de incompatibilidade de enxertia, a diferença de diâmetro no ponto de enxertia, sugerindo, como uma das principais causas dessas diferenças de diâmetro, a migração da seiva a diferentes coeficientes transpiratórios.

\section{CONCLUSÃO}

As combinações Abbè Fetel/Adams e Rocha/Adams são as mais vigorosas em relação às variáveis analisadas, seguidas pela combinação Santa Maria/Adams com vigor intermediário. A combinação Packham's Triumph/EMC não é recomendada, devido ao baixo vigor das plantas com possibilidade de ocorrência de sinais de incompatibilidade morfológica entre a cultivar copa e o portaenxerto.

\section{REFERÊNCIAS}

EPAGRI (EMPRESA DE PESQUISA AGROPECUÁRIA E EXTENSÃO RURAL DE SANTA CATARINA). Dados meteorológicos de São Joaquim e Videira, SC. Florianópolis: EPAGRI/CIRAM/INMET, 2011. Disponível em: <http://ciram. epagri.sc.gov.br/portal/ website/index.jsp?url=jsp/monitoramento/ monitoramento.jsp\&tipo=rios>. Acesso em: 08 jun. 2012.

EBERT, A. et al. First experiences with chill unit's models is southern Brazil. Acta Horticulturae, v.184, p.89-96, 1986. Disponível em: <http://www.actahort.org/books/184/184_8.htm>. Acesso em: 22 ago. 2012.

GIACOBBO, C.L. et al. Compatibilidade entre o marmeleiro portaenxerto cv. 'EMC' e cultivares de pereira. Scientia Agraria, v.8, n.1, p.33-37, 2007. Disponível em: <http://ojs.c3sl.ufpr.br/ojs2/ index.php/agraria/article/view/8339>. Acesso em: 08 jun. 2012.

HARTMANN, H.T. et al. Plant propagation: principles and pratices. New Jersey: Prentice Hall, 2002. 880p. Disponível em: $\quad<\mathrm{http} / /$ www.scielo.br/scielo.php?script=sci nlinks\&ref $=000054 \& \mathrm{pid}=\mathrm{S} 1413-7054200600050003000012 \& \operatorname{lng}=$ en>. Acesso em: 08 jun. 2012.

MILOSEVIC, T.; MILOSEVIC, N. Influence of cultivar and Rootstock on early growth and syllepsis in nursery tress of pear (Pyrus communis L., Rosaceae). Brazilian Archives Biology and Technology, v.54, n.3, p.451456, 2011. Disponível em: <http://www.scielo.br/scielo. php? pid $=$ S 1516-89132011000300004\&script $=$ sci_arttext $>$. Acesso em: 08 jun. 2012. doi: http://dx.doi.org/10.1590/S151689132011000300004.

PIO, R. et al. Grafting of quince 'Portugal' on Cydonia and Chaenomeles rootstocks. Revista Brasileira de Fruticultura, v.30, p.850-852, 2008. Disponível em: <http://www.scielo.br/ scielo.php?pid=S0100-29452008000300051\&script $=$ sci_arttext $>$. Acesso em: 08 jun. 2012. doi: http://dx.doi.org/10.1590/S010029452008000300051

QUEZADA, A.C. et al. (Ed.). Pêra: produção. Pelotas: Embrapa Clima Temperado, 2003. p. 37 - 45. (Frutas do Brasil, 46).

RUFATO, L. et al. Intensidade e época de poda verde em pereira 'Abate Fetel' sobre dois portaenxertos. Revista Brasileira de Fruticultura, v.34, n.2, p.80-85, 2012. Disponível em: <http:// www.scielo.br/scielo.php?script $=$ sci_issuetoc\&pid $=0100$ 294520100050\&lng=pt\&nr $\mathrm{m}=$ iso $>$. Acesso em: 08 jun. 2012. doi: http://dx.doi.org/10.1590/S0100-29452012000200021.

STERN, R. A.; DORON, I. Performance of 'Coscia' pear (Pyrus communis) on nine rootstocks in the north of Israel. Scientia Horticulturae, v.119, p.252-256, 2009. Disponível em: <http:// www.sciencedirect.com/science/article/pii/S0304423808003300>. Acesso em: 08 jun. 2012. doi: http://dx.doi.org/10.1016/j. scienta.2008.08.002.

SANSAVANI, S. et al. Nuovi portinnesti dei peri "William" e "Abate Fétel": confronto fra selezioni di cotogno e franchi clonali. Rivista di Frutticoltura, v.LIX, n.3, p. 31-40, 1997. Disponível em: <http://agris.fao.org/agris-search/search/display.do?f=1998/ IT/IT98013.xml; IT1998061698>. Acesso em: 08 jun. 2012.

SHAFFER, R. et al. Grapevine rootstocks for Oregon vineyards. Oregon: State University, 2004. 11p. Disponível em: <http://www. scielo.br/scielo.php?script $=$ sci_nlinks\&ref $=000123 \&$ pid $=$ S0100$945201100010001400023 \& \operatorname{lng}=$ pt>. Acesso em: 08 Jun. 2012.

TOMAZ, Z.F.P. et al. Compatibilidade de enxertia de cultivares de marmeleiros com pereiras. Revista Brasileira de Fruticultura, v.31,n.4,p.1211-1217, 2009. Disponível em: <http://www.scielo.br/ scielo.php?script $=$ sci_arttext\&pid=S0100-29452009000400041>. Acesso em: 08 jun. 2012. doi: http://dx.doi.org/10.1590/S010029452009000400041

VALLI, R. Arboricoltura: generale e speciale. 6.ed. DELL'EMILIA, O. (Bo): CALDERINI EDAGRICOLE, 2002. 658p. 